\title{
Antimicrobial activity of Tephrosia purpurea (Linn.) Pers. and Mimusops elengi (Linn.) against some clinical bacterial isolates
}

\author{
B.N.L.D. Rangama ${ }^{1}$, C.L. Abayasekara ${ }^{1 *}$, G.J. Panagoda ${ }^{2}$ and M.R.D.M. Senanayake ${ }^{2}$ \\ ${ }^{1}$ Department of Botany, Faculty of Science, University of Peradeniya, Peradeniya. \\ ${ }^{2}$ Division of Microbiology, Faculty of Dental Sciences, University of Peradeniya, Peradeniya.
}

\begin{abstract}
Plants used in traditional medicinal systems have proved to be reliable sources of antibmicrobial compounds. Two native plants, Tephrosia purpurea (Linn.) Pers. (Fabaceae) and Mimusops elengi (Linn.) (Sapotaceae) were screened for their antimicrobial activity. Preliminary testing of antimicrobial activity of T. purpurea against 3 standard cultures (Staphylococcus aureus [NCTC 6571], Pseudomonas aeruginosa [NCTC 10662], E. coli [NCTC 10418] and one clinical isolate of Candida spp. was performed with water extracts of leaves, pods and roots using the 'disc diffusion bioassay'. Subsequently, the antimicrobial activity of ethanolic root and leaf extracts against the above three standard isolates and clinical isolates of two strains of Staphylococcus, two strains of Pseudomonas and nine coliforms were tested using the 'well method'. The active extracts were subjected to the Minimum Inhibitory Concentration (MIC) agar dilution method, to determine the minimum inhibitory concentration of each extract. Further, the effect of plant maturity was tested on the antimicrobial activity of T. purpurea. In addition, ethonolic extracts were prepared from the bark of $M$. elengi and tested for its antimicrobial activity against the above bacterial isolates.
\end{abstract}

Ethanolic root extracts of T. purpurea were found to be active against $P$. aeruginosa, two other Pseudomonas strains and two coliform strains. Ethanolic leaf extracts and all the water extracts showed no activity against any of the isolates. The bark extract of $M$. elengi showed activity against three Staphylococcus isolates including S. aureus. The MIC of ethanolic root extracts of T. purpurea and bark extract of M. elengi were both found to be $128 \mathrm{mg} / \mathrm{L}$. There were no differences between the antimicrobial activities of the extracts of T. purpurea plants at different maturity levels.

Keywords: Antimicrobial activity, coliforms, Mimusops elengi, Pseudomonas aeruginosa, Staphylococcus aureus, Tephrosia purpurea.

\section{INTRODUCTION}

Herbal medicines are employed in a wide variety of health related applications ranging from treatment of common colds to treatment of cancer ${ }^{1}$. Encouraging results from research in the recent past on medicinal properties of plants, have led to scientists searching for plant derived drugs. Plant species used in various traditional medicinal systems have been reliable and convenient sources to select plants to be analysed for medicinal properties ${ }^{2}$.

Various chemical compounds synthesised in plants give them their medicinal properties. Lately, higher plants have been screened extensively for antimicrobial compounds $\mathrm{s}^{1-4}$. Antimicrobial compounds can be broadly defined as compounds capable of destroying or inhibiting the growth of microorganisms.

T. purpurea (Linn.) Pers (Fabaceae) is a perennial herb which is known as pila in Sinhala, kolinji in Tamil, wild indigo in English, and Sharpunkha in Sanskrit. This plant shows a pantropic distribution. It is common in India, Sri Lanka, Malay Peninsula, China and Hawaii. In Sri Lanka, it is more common in the low country, in both wet and dry zones ${ }^{5}$. T. purpurea grows up to a height of about $30-60 \mathrm{~cm}$. This plant is extensively exploited as a medicinal plant. In the Ayurveda system, T. purpurea is referred to as Sarwa wran vishapaha which implies that it can heal any type of wound. It is also believed to be very effective in the treatment of inflammation and enlargement of spleen and liver, therefore, it is also called plihāri or plihaśathru $(\text { pliha }=\text { spleen })^{6}$. The roots and sometimes the whole plants are used in medicine ${ }^{7}$. 
A decoction of roots is used to treat dyspepsia, cough, asthma, fever, ulcers, skin diseases, flatulence, colic, and as a blood purifier and an anthelmintic. Wound healing potential of T. purpurea has also been identified ${ }^{8}$.

M. elengi (Linn.) (Sapotaceae) is a perennial tree distributed in India, Sri Lanka, Malay Peninsula, and Andaman Islands ${ }^{5}$. The plant is commonly known as Munamal in Sinhala, Makil in Tamil, Spanish Cherry in English and Sinha kesara in Sanskrit, and is widely used in traditional medicinal systems. A decoction is prepared from the bark, which can be used to treat diseases of the gum and teeth. It is also used to treat the diseases of the bladder and urethra. It gives relief from fever, and increases female fertility ${ }^{7}$. Flowers and fruits are used to prepare lotions for the treatment of sores. Anti-ulcer activity ${ }^{9}$ and hypotensive activity ${ }^{10}$ have been detected in the bark extract of Mimusops. Leaves have shown antibacterial activity ${ }^{4}$.

The current study was designed to determine the antimicrobial activity of $T$. purpurea and $M$. elengi on some bacterial isolates. Further, the effect of maturity of the plants on the antimicrobial activity of $T$. purpurea was also investigated.

\section{METHODS AND MATERIALS}

Microbial strains: Three standard cultures (Staphylococcus aureus [NCTC 6571], Pseudomonas aeruginosa [NCTC 10662], E. coli [NCTC 10418]) and clinical bacterial isolates (fixed cultures on sterile filter paper strips, which were stored in the freezer at $-70{ }^{\circ} \mathrm{C}$ ) from various tissue samples of patients received at the Microbiology Laboratory of the Faculty of Dental Sciences, University of Peradeniya, were used. The clinical isolates included, 2 strains of Staphylococcus, 2 strains of Pseudomonas and 9 strains of coliforms. Bacterial filter paper strips were pressed on different media, in order to revive cultures, viz. Staphylococcus strains on solidified Blood Agar and all the other isolates on solidified Mac Conkey Agar. The plates were incubated at $37^{\circ} \mathrm{C}$ for $24 \mathrm{~h}$. The resulting colonies of the clinical isolates were tested for the confirmation of their identity by performing the Coagulase test for Staphylococcus strains and the Cytochrome Oxidase test for Pseudomonas strains ${ }^{11}$. In addition to the above, one Candida isolate was also used.

\section{Plant material:}

a) T. purpurea samples for the preparation of water extracts: Mature plants of T. purpurea growing along the roadside of Kuliyapitiya (Kurunegala District) were uprooted carefully. They were washed well with water to remove dirt and debris.

b) T. purpurea samples for the preparation of ethanolic extracts: Mature seeds of $T$. purpurea were obtained from plants growing along the roadside of Kuliyapitiya (Kurunegala District). The seeds were sown in a seed bed, at the Agricultural farm at Meewathura (Kandy District). The seed bed was watered regularly, until the seedlings were 3 wks old. A soil mixture was prepared by mixing loamy soil from the Agricultural farm at Meewathura, with sand and coir dust in a 3:1:1 ratio and 40 cement pots (diameter: $30 \mathrm{~cm}$, height: $22 \mathrm{~cm}$ ) were filled with the prepared soil mixture. Two to three wks old Tephrosia seedlings from the seed bed were transferred into each pot. The pots were kept in a plant house. They were watered daily with $2 \mathrm{~L}$ of water per pot.

Harvesting of T. purpurea was done at two different stages of maturity; viz 3 months and 5 months; (before and after flowering and fruit set respectively). At each harvesting stage, 20 plants were uprooted and washed well with water to remove the soil.

c) M. elengi bark samples for the preparation of ethanolic extracts: Bark of a mature $M$. elengi tree, from Kuliyapitiya (intermediate zone), was peeled using a knife, and taken to the laboratory. Bark segments were cut into pieces $(1 \mathrm{~cm} \mathrm{x} 0.5 \mathrm{~cm})$.

\section{Preparation of extracts:}

a) Water extracts for preliminary testing of T. purpurea: Mature leaves, pods and roots of freshly harvested plants were separated. Samples of $20 \mathrm{~g}$ each were taken from the above 3 plant parts and from a combination of the above. Each sample was boiled in $80 \mathrm{~mL}$ of distilled water and a final volume of $10 \mathrm{~mL}$ was obtained by evaporation of water, according to Ayurvedic practice. The extracts were filtered using Whatman No.1 filter paper, and transferred into sterile glass vials.

b) Ethanolic extracts of $T$. purpurea: Preparation of ethanolic extracts from 3 month old and 5 month old Tephrosia plants was done separately. Harvested T. purpurea plants were washed again with water, and allowed to dry at room temperature $\left(27 \pm 1^{\circ} \mathrm{C}\right)$. The roots were separated from the plants. Leaves were picked separately. The separated plant parts of Tephrosia were cut into small pieces $(1 \mathrm{~cm} \times 0.5 \mathrm{~cm})$. Fifty grams from each sample were soaked in $250 \mathrm{~mL}$ of $99 \%$ ethanol for $24 \mathrm{~h}$ at room temperature $\left(27 \pm 1^{\circ} \mathrm{C}\right)$. The plant parts were subjected to the vacuum infiltration technique 
with the same aliquot of ethanol at room temperature for $1 \mathrm{~h}^{\mathbf{1 2}}$. The resulting ethanolic extract was filtered using Whatman No.1 filter paper. This was repeated with 3 changes of ethanol for Tephrosia plant parts and 4 changes for Mimusops bark $^{\mathbf{1 3}}$. The ethanolic filtrates were concentrated at $40^{\circ} \mathrm{C}$ using a rotary evaporator to obtain extracts devoid of ethanol. They were freeze-dried and transferred into sterile glass vials, and were stored in the freezer, at $-70^{\circ} \mathrm{C}$.

c) Ethanolic extracts of $M$. elengi: Fifty gram samples were subjected to the above method of extraction using ethanol. The extracts were evaporated and freeze-dried and stored until further use as for Tephrosia.

\section{Bioassays:}

a) Disc diffusion bioassay with water extracts of T. purpurea: Broth cultures were prepared by inoculating, isolated pure standard cultures of $S$. aureus [NCTC 6571], P. aeruginosa [NCTC 10662], E. coli [NCTC 10418] and Candida spp. to Brain Heart Infusion Broth (BHIB) and incubating at $37^{\circ} \mathrm{C}$ overnight.

$20 \mathrm{~mL}$ of sterile molten Mueller Hinton Agar (MHA) at $45{ }^{\circ} \mathrm{C}$ was pipetted out into a sterile Universal bottle, to which $50 \mu \mathrm{L}$ of one of the prepared broth cultures was added and the bottle was capped. The contents were mixed thoroughly, poured immediately into a sterile Petri dish, and allowed to set. Subsequently, each plate was marked into 5 partitions on the reverse side. The partitions were labeled as L (leaves), P (pods), R (roots), C (combination) and DW (distilled water). This procedure was repeated with all 4 microbial isolates in triplicate. Antibiotic testing filter paper discs $(6 \mathrm{~mm}$ in diameter) were saturated with the prepared water extracts of T. purpurea (about $150 \mu \mathrm{L}$ ), by placing a drop at a time on the disc using a micropipette and allowing it to absorb. A control disc was prepared using distilled water. The discs were placed on the corresponding labeled partition of each Perti dish and incubated at $37^{\circ} \mathrm{C}$ overnight, and the zone of inhibition around each disc was measured after 18-24 hrs.

b) 'Agar diffusion' bioassay with ethonolic extracts of T. purpurea and M. elengi: Tephrosia root extracts-3 months old (R1), 5 months old (R2), Tephrosia leaf extracts-5 months old (L), and Mimusops bark extract (M) were tested using the 'Agar diffusion' bioassay for their antimicrobial activity.

Molten Mueller Hinton Agar (MHA) was poured into 16 sterile $90 \mathrm{~mm}$ Petri dishes (15 mL per dish) and allowed to solidify. Equal volumes $(0.25 \mathrm{~mL})$ of each broth bacterial culture (McFarland series), was pipetted on to an MHA plate separately, and spread with a sterile glass spreader. They were allowed to dry at $44{ }^{\circ} \mathrm{C}$ for $10 \mathrm{~min}$. Using a sterile $8 \mathrm{~mm}$ cork borer, wells were cut on each MHA plate. The wells were named with the code names of the four extracts (R1, R2, L, and M) and as SDW (sterile distilled water) and D (Dimethylsulfoxide - DMSO).

$30 \mathrm{mg}$ of each of the freeze dried leaf extracts and the bark extract was dissolved in sterile distilled water, while similar quantities of the root extracts were dissolved in DMSO (since the root extracts were not completely soluble in water). These solutions were then loaded into the corresponding wells of each Petri dish, using a micropipette. The wells named as $\mathrm{W}$ and $\mathrm{D}$ were loaded with sterile distilled water and DMSO respectively. The plates were subsequently incubated at $37^{\circ} \mathrm{C}$ overnight, and the zone of inhibition around each well was measured. This procedure was repeated 3 times.

\section{Determination of the Minimum Inhibitory Concentration (MIC) of antimicrobial extracts:}

a) Preparation of bacterial cultures with similar cell densities: Extracts which showed significant antimicrobial activity at the activity test were tested for their MIC. Broth cultures of bacteria with their turbidity equal to that of the standard McFarland 0.5 solution were prepared as follows.

The isolated bacterial colonies were streaked on their respective media and incubated at $37^{\circ} \mathrm{C}$ overnight. A loopful from an isolated colony was taken to a sterile inoculating needle. It was smeared on the inner wall of a sterile screw-cap tube, containing $10 \mathrm{~mL}$ of sterile normal Saline. Subsequently, the tube was capped and vortexed for several seconds to dissolve the bacterial culture well. The turbidity of the broth culture obtained was made similar to that of the McFarland 0.5 standard solution by dissolving more of the colony or diluting with more normal Saline. This was repeated with all the bacterial isolates, to obtain broth cultures with more or less similar cell densities and stored under refrigeration $\left(4^{\circ} \mathrm{C}\right)$ for use on the same day.

A concentration series of the extract was prepared ${ }^{14}$ by pipetting out different volumes from the R1 stock solution into 8 sterile Universal bottles as shown in Table 1. This procedure was repeated for all other extracts.

$20 \mathrm{~mL}$ of molten $\mathrm{MHA}\left(\right.$ at $50^{\circ} \mathrm{C}$ ) was transferred into a Universal bottle with an extract concentration of 128 $\mathrm{mg} / \mathrm{L}$, using a sterile pipette and a dispenser. The bottles were capped and the contents were mixed thoroughly. 
Subsequently, the medium was poured into a sterile $90 \mathrm{~mm}$ Petri dish which was marked into 16 partitions on the reverse side. The partitions were labeled with the names of the bacterial isolates. This was repeated with the remaining 8 concentrations (ranging from 0-64 mg/L) in universal bottles. MHA was allowed to set and the plates were dried at $44^{\circ} \mathrm{C}$. A $10 \mu \mathrm{L}$ drop each from the prepared broth bacterial cultures was placed on agar on the corresponding partition using a micropipette. After absorption of culture, they were incubated at $37^{\circ} \mathrm{C}$ overnight.

The entire procedure was repeated for the remaining active extracts in triplicate.

\section{RESULTS}

\section{Activity test of water extracts-disc diffusion bioassay}

The zones of inhibition observed in the disc diffusion bioassay are shown in the Table 2 .

Since the average zones of inhibition were $<3 \mathrm{~mm}$, it could be concluded that the water extracts of $T$. purpurea had no antibiotic activity on $S$. aureus, P. aeruginosa, E. coli, and Candida spp.

Table 1: Volumes of the stock solution and concentration of the final solution of extract

\begin{tabular}{cc}
\hline $\begin{array}{c}\text { Volume of stock solution } \\
(\mu \mathrm{L})\end{array}$ & $\begin{array}{c}\text { Concentration of the final solution } \\
(\mathrm{mg} / \mathrm{L})\end{array}$ \\
\hline 2560 & 128 \\
1280 & 64 \\
640 & 32 \\
320 & 16 \\
160 & 8 \\
80 & 4 \\
40 & 2 \\
20 & 1 \\
0 & 0 \\
\hline
\end{tabular}

\section{Activity test of ethanolic extracts-Agar diffusion bioassay}

The zones of inhibition observed in the 'Agar diffusion bioassay' of the ethanolic extracts are shown in Table 3. Zone of inhibition $>3 \mathrm{~mm}$ were considered as inhibitions resulting from considerable antimicrobial activity.

The bark extract of $M$. elengi (M) showed considerable inhibition of the three Staphylococcus isolates i.e. S. aureus [NCTC 6571] (Table 3 and Figure 1), Staphylococcus strain 1 and Staphylococcus strain 2. There was no difference between the average inhibition zones within these three isolates. No inhibition was observed on the other isolates, viz: coliforms and Pseudomonas spp.

The root extracts of T. purpurea (R1 and R2) showed considerable inhibition of the three Pseudomonas isolates i.e. P. aeruginosa [NCTC 10662] (Table 3 and Figure 2), Pseudomonas strain 1, Pseudomonas strain 2 and two of the coliform strains i.e. coliform strain 6 and coliform strain 9. There was no difference between the average inhibition zones given by Tephrosia root extract within the three Pseudomonas isolates and within coliform strains 6 and 9. No inhibition was shown by the leaf extract (L) on any of the isolates tested (Figure 2).

DMSO showed a very slight inhibition on the three Pseudomonas strains and the ten coliform strains (including E. coli). However, it was almost insignificant when compared with the inhibition by the root extracts. No inhibition was shown by DMSO on any of the Staphylococcus strains used.

\section{Minimum Inhibitory Concentrations (MIC)}

The inhibition of Staphylococcus strains was observed only in the plates containing a concentration of $128 \mathrm{mg} / \mathrm{L}$ of Mimusops bark extract, i.e. the highest concentration used (Figure 3). Similarly, the inhibition of Pseudomonas

Table 2: Average zones of inhibition given by the antibiotic discs soaked with water extracts of T. purpurea

\begin{tabular}{lccccc}
\hline & \multicolumn{5}{c}{ Average zone of inhibition (mm) } \\
\cline { 2 - 5 } Isolate & \multicolumn{5}{c}{ Extract } \\
\cline { 2 - 5 } & Root & Leaves & Pods & Combination & \\
\hline S. aureus [NCTC 6571] & 0.0 & 0.0 & 0.0 & 0.0 & 0.0 \\
P. aeruginosa [NCTC 10662] & 1.0 & 0.0 & 0.0 & 1.0 & 0.0 \\
E. coli [NCTC 10418] & 1.0 & 0.0 & 0.0 & 1.0 & 0.0 \\
Candida spp. & 0.0 & 0.0 & 0.0 & 0.0 & 0.0 \\
\hline
\end{tabular}


strains and the coliform strains 6 and 9 was observed in the plates containing a concentration of $128 \mathrm{mg} / \mathrm{L}$ Tephrosia root extracts. There was no inhibition of any of the isolates tested in concentrations lower than $128 \mathrm{mg} / \mathrm{L}$.

Table 3: Average zones of inhibition given by ethanolic extracts of T. purpurea and M. elengi

\begin{tabular}{|c|c|c|c|c|c|c|}
\hline \multirow{3}{*}{ Isolate } & \multicolumn{6}{|c|}{ Average zone of inhibition (mm) } \\
\hline & \multicolumn{6}{|c|}{ Extract } \\
\hline & $\mathrm{R} 1$ & R2 & $\mathrm{L}$ & M & $\mathrm{D}$ & $\mathrm{W}$ \\
\hline S. aureus [NCTC 6571] & 0 & 0 & 0 & 6.5 & 0 & 0 \\
\hline Staphylococcus 1 & 0 & 0 & 0 & 6.5 & 0 & 0 \\
\hline Staphylococcus 2 & 0 & 0 & 0 & 6.5 & 0 & 0 \\
\hline P. aeruginosa & 4.5 & 4.5 & 0 & 0 & $<1$ & 0 \\
\hline Pseudomonas 1 & 4.5 & 4.5 & 0 & 0 & $<1$ & 0 \\
\hline Pseudomonas 2 & 4.5 & 4.5 & 0 & 0 & $<1$ & 0 \\
\hline E. coli [NCTC 10418] & 0 & 0 & 0 & 0 & 1 & 0 \\
\hline Coliform 1 & 0 & 0 & 0 & 0 & $<1$ & 0 \\
\hline Coliform 2 & 0 & 0 & 0 & 0 & $<1$ & 0 \\
\hline Coliform 3 & 0 & 0 & 0 & 0 & $<1$ & 0 \\
\hline Coliform 4 & 0 & 0 & 0 & 0 & $<1$ & 0 \\
\hline Coliform 5 & 0 & 0 & 0 & 0 & $<1$ & 0 \\
\hline Coliform 6 & 5.0 & 5.0 & 0 & 0 & $<1$ & 0 \\
\hline Coliform 7 & 0 & 0 & 0 & 0 & $<1$ & 0 \\
\hline Coliform 8 & 0 & 0 & 0 & 0 & $<1$ & 0 \\
\hline Coliform 9 & 4.5 & 4.5 & 0 & 0 & 1 & 0 \\
\hline
\end{tabular}

(R1)-Tephrosia 3 months old root extract, (R2)-Tephrosia 5 months old root extract, (L)-Tephrosia -5 months old leaf extract, and (M) Mimusops bark extract. (D) DMSO and (W) Sterile distilled water

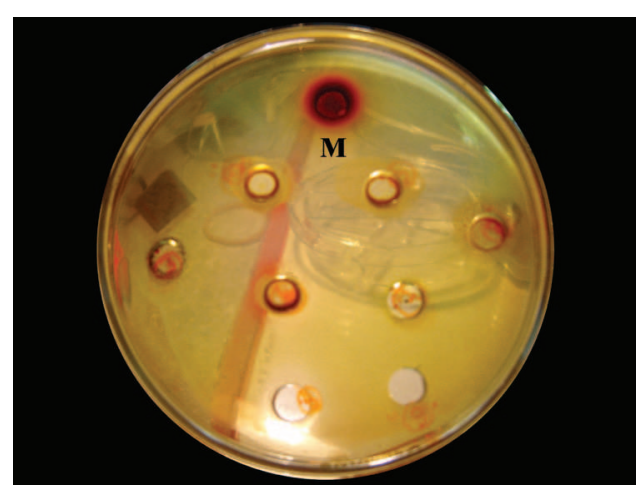

Figure 1: Inhibition of S. aureus [NCTC 6571] around the well, loaded with the bark extract of Mimusops (M) - The transparent concentric area around the well signifies the absence of bacterial growth. Brick colour around the well is due to the diffused extract. The remaining wells which were loaded with the extracts of Tephrosia and the two controls show no inhibition.

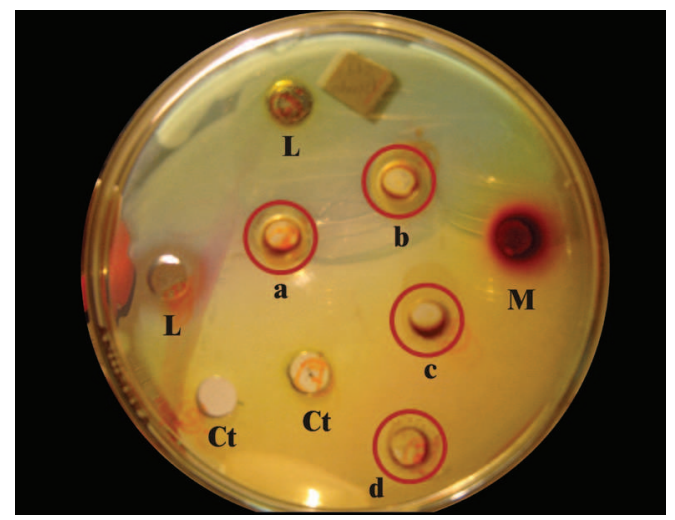

Figure 2: Inhibition of $P$. aeruginosa [NCTC 10662] by the root extracts of $T$. purpurea. [ a, b, c and d marked in red circles are wells loaded with the root extracts]. The remaining wells (L) which were loaded with the leaf extracts of Tephrosia, bark extract of Mimusops (M) and the two controls (Ct) show no inhibition. 


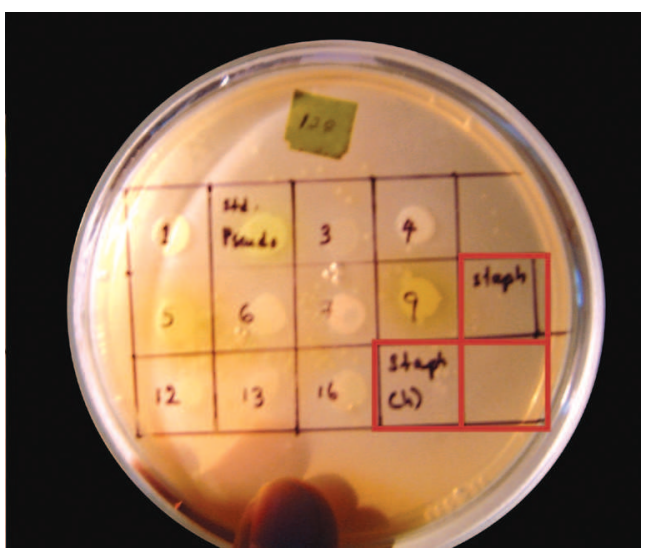

Figure 3: Plate containing $128 \mathrm{mg} / \mathrm{L}$ of Mimusops bark extract, showing absence of growth of three Staphylococcus strains on partitions marked in red. Other partitions containing all other isolates indicate growth.

\section{DISCUSSION}

In the ayurvedic and the traditional medicinal systems of Sri Lanka, the roots of T. purpurea are used for the treatment of the diseases of gum, spleen, liver and skin. In both medicinal systems, the decoction is prepared by boiling the roots in water. However, according to the test results, the water extract of roots, leaves and pods of $T$. purpurea did not seem to possess considerable antimicrobial properties on any of the pathogens tested. The belief is that it is not the antimicrobial property of Tephrosia which is employed in traditional medicine, rather the astringent, antipyretic, anthelmintic, diuretic and laxative properties (Harischandra Wijesinghe, personal communication).

However, the ethanolic root extracts showed significant antimicrobial activity against the three Pseudomonas strains. The three strains of the coliforms tested were not inhibited by the water extract. The reason for the water extract not to be effective could be due to the heat sensitivity of the antibiotic compound, which gets destroyed during the process of boiling. Another possible reason is that the antibiotic compound is more soluble in alcohol than in water (due to higher polarity). This is supported by the fact that there was a minute zone of inhibition on both Pseudomonas and E. coli even with the water extract of the roots.

The inhibition of Pseudomonas and coliforms by the Tephrosia ethanolic root extract was however less than the inhibition of Staphylococcus by the Mimusops extract. A considerable activity of both aqueous and alcoholic extracts of $M$. elengi against several bacterial strains including $S$. aureus has been revealed ${ }^{4}$. Here, the activity of the water extract has been shown to be greater than that of the ethanolic extract against Alcaligenes faecalis. The significant antibiotic activity shown by Mimusops bark extract against three Staphylococcus strains in the current study may bring a ray of hope for MRSA (Methicillin-Resistant Staphylococcus aureus) infections. Further studies will have to be carried out with the purified active compound and tested on MRSA strains for antimicrobial activity. In a similar study plant extracts of Bidens pilosa, Bixa orellana, Cecropia peltata, Cinchona officinalis, Gliricidia sepium, Jacaranda mimosifolia, Justicia secunda, Piper pulchrum, Polygala paniculata and Spilanthes americana antimicrobial activity against various pathogens including $S$. aureus, E. coli and Candida spp. has been shown. However, the extracts have been ineffective against $P$. aeruginosa and Streptococcus spp. ${ }^{15}$ Literature cites antimicrobial action of T. purpurea against acne-inducing Propionibacterium acnes and Staphylococcus epidermidis ${ }^{1}$. However, the MIC values obtained, are much higher than the values obtained in the current study, viz $675 \mathrm{mg} / \mathrm{L}$ and 2500 $\mathrm{mg} / \mathrm{L}$ respectively.

The MIC, of all the extracts exhibiting antimicrobial activity were found to be $128 \mathrm{mg} / \mathrm{L}$; i.e. the highest concentration used. However, this value was obtained for crude extracts. For more accurate determination of the MIC, the active compound has to be isolated. A much lower MIC can be expected in such purified extracts. The MIC of Ciprofloxacin on P. aeruginosa (ATCC 27853) is $0.25 \mathrm{mg} / \mathrm{L}$ and that of Vancomycin on $S$. aureus (ATCC 25923 ) is $0.5 \mathrm{mg} / \mathrm{L}^{14}$. It is reported by Abu-Shanab et al. that the crude extracts of different plants (Althaea officinalis, Mentha longifolia, Melissa officinalis, Rosa damascena) showed antibiotic activity against MRSA with the most potent extract with an MIC in the range of 395 to $780 \mathrm{mg} / \mathrm{L}^{16}$. Accordingly the results of this study indicate that $T$. perpurea and $M$. elengi extracts have considerable promise to be used as antimicrobial agents. T. purpurea and M. elengi could be used to isolate new bio-active natural products that may lead to the development of new pharmaceuticals that address therapeutic needs. For the exploitation of the antimicrobial activity of these extracts, the isolation and chemical characterisation of these compounds need to be carried out. Subsequent to such studies the extracts of T. purpurea and M. elengi could be incorporated into products such as tablets, other forms of drugs, disinfectants, tooth paste etc. 


\section{Acknowledgment}

The assistance given by Mr. H.N. Fernando of the Department of Microbiology, Faculty of Dental Sciences, University of Peradeniya is gratefully acknowledged.

\section{References}

1. Kumar G.S., Jayaveera K.N., Sanjay U.P., Swamy B.M.V. \& Kumar D.V.K. (2007). Antimicrobial effects of Indian medicinal plants against acne-inducing bacteria. Tropical Journal of Pharmaceutical Research 6(2): 717-723.

2. Nair R., Kalariya T. \& Chanda S. (2005). Antibacterial activity of some selected indian medicinal flora. Turkish Journal of Biology 29(1): 41-47.

3. Harris C.S., Rajakaruna N. \& Towers G.H.N. (2002). Antimicrobial activity of plants collected from serpentine outcrops in Sri Lanka. Pharmaceutical Biology 40(3): 235-244.

4. Nair R. \& Chanda S. (2007). Antibacterial activities of some medicinal plants of the Western Indian region of India. Turkish Journal of Biology 31: 231-236.

5. Jayaweera D.M.A. (1981-1982). Medicinal Plants Used in Ceylon. Vol. 3 \& 5. pp. 66-67 \& 248-249. National Science Council of Sri Lanka, 47/5, Maitland Place, Colombo.

6. Sivarajan V.V. \& Balachandran I. (1993). Ayurvedic Drugs and Their Plant Sources. p. 433. Oxford and IBH Publishing Co. Pvt. Ltd, New Delhi, India.

7. Joy P.P., Thomas J., Mathew S. \& Skaria B.P. (1998). Medicinal Plants. p. 210. Kerala Agricultural University, Kerala, India.

8. Lodhi S., Pawar R.S., Jain A.P. \& Singhai A.K. (2006). Wound healing potential of Tephrosia purpurea (Linn.)
Pers. in rats. Journal of Ethnopharmacology 108(2): 204210.

9. Gandhi M.S., Goswami S.S., Santani D., Shah M.B. \& Shah P.J. (2003). Study of Mimusops elengi bark in experimental gastric ulcers. Journal of Ethnopharmacology 89(2-3): 305-311.

10. Dar D. \& Agarwal V.S. (1991). Fruit Drug Plants of India, $1^{\text {st }}$ Edition, pp. 128-129. Kalyani Publishers, New Delhi, India.

11. Cheesbrough M. (1998). District Laboratory Practice in Tropical Countries - Part 2, p. 434. Cambridge University Press, Cambridge, UK.

12. Adikaram N.K.B. \& Bandara B.M.R. (1998). Methodology for studying defense mechanisms against fungal pathogens: an overview. In: Disease Resistance in Fruits (Eds. G.I. Johnson, E. Highley \& D.C. Joyce). ACIAR Proceedings, Canberra, Australia 80: 177-185.

13. Abayasekara C.L. (1998). Freckle disease (Phyllosticta musarum) in Banana: biology, control and host defense responses. Ph.D Thesis, University of Peradeniya, Peradeniya.

14. Andrews J.M. (2001). Determination of minimum inhibitory concentrations. Journal of Antimicrobial Chemotherapy 48: 5-16.

15. Rojas J.J., Ochoa V.J., Ocampo S.A. \& Muñoz J.F. (2006). Screening for antimicrobial activity of ten medicinal plants used in Colombian folkloric medicine: a possible alternative in the treatment of non-nosocomial infections. BMC Complementary and Alternative Medicine 6(2). doi: 10.1186/1472-6882-6-2.

16. Abu-shanab B., Adwan G., Jarrar N., Abu-hijleh A. \& Adwan K. (2006). Antibacterial activity of four plant extracts used in Palestine in folkloric medicine against methicillin-resistant Staphylococcus aureus. Turkish Journal of Biology 30: 195-198. 